\title{
Investigation of dimensional accuracy in incremental sheet metal hammering process: a parametric study
}

\author{
A. Asgari ${ }^{a}$, M. Sedighi And M. Riahi \\ School of Mechanical Engineering, Iran University of Science and Technology, 16846 Narmak, Tehran, Iran
}

Received 17 June 2014, Accepted 9 February 2015

\begin{abstract}
With the increasing demand for low-volume production, the incremental forming process is developed as a rapid prototyping method. Incremental sheet metal hammering method has been used in recent years. In this process, a sequence hammer moving over a clamped Aluminum sheet and a three dimensional workpiece can be produced by using a simple die. In this paper, the influence of the punch diameter on the dimensional accuracy was studied. This component was divided to four sections: thickness, overall geometry, surface quality and spring-back. By using the image processing and roughness tester, the values of results were obtained and analyzed. The results had revealed that the surface roughness and spring-back were decreased by decreasing the punch diameter. Overall geometry was deviated from the ideal shape by increasing the punch diameter.
\end{abstract}

Key words: Incremental forming / Punch diameter / dimensional accuracy / aluminum sheet

\section{Nomenclature}

\begin{tabular}{|lll|}
\hline$f$ & Frequency of punch & $\mathrm{Hz}$ \\
$\mathrm{d} x$ & Feed-rate in $x$ direction & $\mathrm{mm} \cdot \mathrm{s}^{-1}$ \\
$\mathrm{~d} y$ & Feed-rate in $y$ direction & $\mathrm{mm} \cdot \mathrm{s}^{-1}$ \\
$t$ & Final thickness & $\mathrm{mm}$ \\
$t_{0}$ & Initial thickness & $\mathrm{mm}$ \\
$h$ & Cone height & $\mathrm{mm}$ \\
$D_{0}$ & Large diameter of cone & $\mathrm{mm}$ \\
$\alpha$ & Forming angle & degree \\
$f_{\mathrm{h}}$ & Horizontal feed & $\mathrm{mm}$ \\
$f_{\mathrm{v}}$ & Vertical feed & $\mathrm{mm}$ \\
$f_{\mathrm{t}}$ & Total feed & $\mathrm{mm}$ \\
$R_{\mathrm{p}}$ & Punch radius & $\mathrm{mm}$ \\
$R_{\mathrm{th}}$ & Theoretical roughness & $\mu \mathrm{m}$ \\
$R_{\mathrm{s}}$ & Initial curvature of sheet & $\mathrm{mm}$ \\
$D$ & Punch diameter & $\mathrm{mm}$ \\
$\Delta x$ & Spring-back in $x$ direction & $\mathrm{mm}$ \\
$\Delta y$ & Spring-back in $y$ direction & $\mathrm{mm}$ \\
\hline
\end{tabular}

\section{Introduction}

Nakajima [1] studied some primary concepts of incremental forming as a flexible process by numerical control (NC) machines. Allwood and Utsunomia [2] later pointed

\footnotetext{
a Corresponding author:

a_asgari@mecheng.iust.ac.ir
}

out many advantages of the incremental forming process. In recent years, evaluation of its parameters effect was considered. For example, Ambrogio et al. [3] presented investigation of the parameters on the dimensional accuracy in incremental forming. Micari et al. [4] pointed out geometrical shape, dimensional accuracy and method improvement. Han et al. [5] used the image processing method by laser to predict the spring back. Ham and Jeswiet [6] introduced a method based on Box-Behnken design analysis for improvement of dimensional accuracy in single point incremental forming.

Incremental sheet metal hammering (ISMH) has been developed in recent years. This process is based on the application of continuous impacts on the clamped sheet. Overall view of this process is shown in Figure 1. Some limited research activities have been reported on this process. Puzik [7] designed and made several impacting devices. He also presented several methods to reduce forming and friction forces. Schafer [8] introduced a type of rapid prototyping process using a hammering tool. Tanaka et al. [9] presented a method of error debugging by introducing an impact device with servo-motor. Luo et al. [10] studied a new impact incremental forming system by modeling and simulation. Furthermore, Luo et al. [10], completed their studies by making a prototype of impact forming machine with hydraulic mechanism. They also presented the results of theoretical and experimental studies and identified the process effective parameters. 
1. Clamp

2. Initial shape

3. Punch

4. Final shape

5. Support

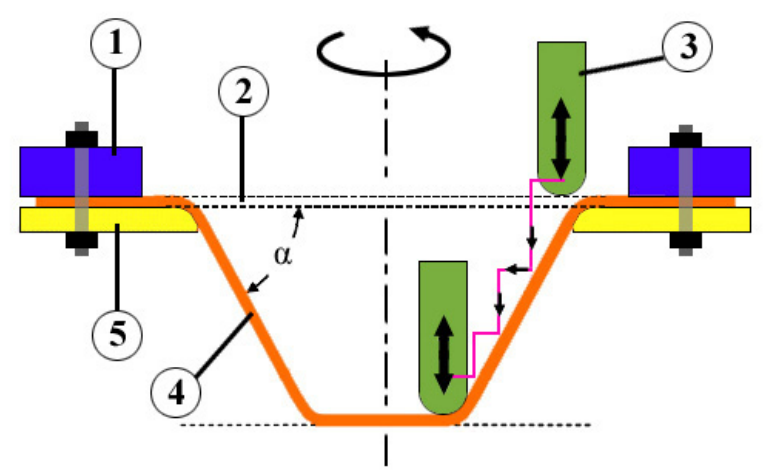

Fig. 1. Overall view of incremental sheet metal hammering.

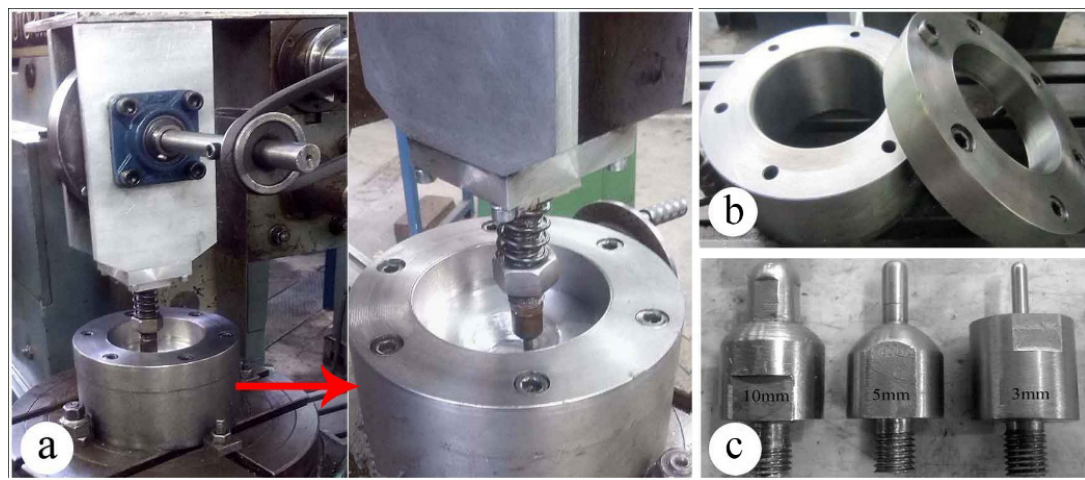

Fig. 2. View of experimental devices, (a) hammering tool, (b) clamping system, (c) punch head.

In this research, the effect of punch diameter on dimensional accuracy in incremental sheet metal hammering process has been studied. Firstly, the aluminum sheet was clamped and formed under successive blows of punch. Then, the samples are provided for image processing and roughness testing. Finally, from the obtained results, the influence of the punch diameter on the final thickness, overall geometry, surface roughness and the spring-back was studied.

\section{Experiment}

This section introduces the materials, procedure and dimensional evaluation of ISMH process.

\subsection{Material}

The Al-1100-O sheet was used as a material with an initial thickness of $0.5 \mathrm{~mm}$ and the blank diameter is $140 \mathrm{~mm}$.

\subsection{Procedure}

Initially, the sheet boundary was fixed by clamping. Then, the punch with a certain diameter was placed on the hammer. By applying the successive blows of the punch on the clamped sheet, the ISMH process has been accomplished (Figs. 2a and $2 \mathrm{~b}$ ). The process was done by different punch diameters (Fig. 2c). The strategy of sheet forming includes two stages: decreasing cone radius, and, increasing depth. The hammering frequency is equal to $25 \mathrm{~Hz}$, the cone angle is $45^{\circ}$ and hammering increment is equal to $0.1 \mathrm{~mm}$. Forming strategy and definition of the cone shape are shown in Figure 3.

\subsection{Methodology for dimensional accuracy study}

This section describes the methods for evaluation of the dimensional accuracy in ISMH process that included: final thickness, overall geometry, surface quality and the spring-back.

\subsubsection{Assessment of final thickness}

As introduced, the sample was formed to a cone shape. The results of samples thickness in the cross-section are obtained versus punch diameter by cutting the formed samples with wire-cut machine and image processing.

In order to predict the final thickness after forming process, the cosine law can be used (Fig. 6). This law is based on simple geometric relationship. In the cosine law, $\alpha$ is the cone angle, $t_{0}$ is the initial thickness and $t$ is the final thickness. The result will be presented in Section 3.1. 


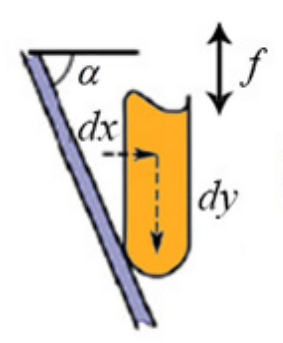

a

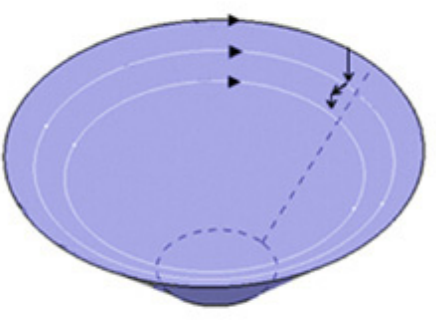

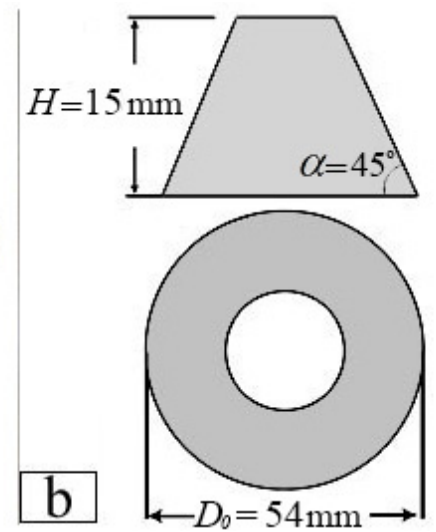

Fig. 3. The schematic of the forming strategy, (a) forming parameters $\left(\mathrm{d} x=\mathrm{d} y=0.1 \mathrm{~mm} \cdot \mathrm{s}^{-1}\right.$ ), (b) definition of the cone shape.

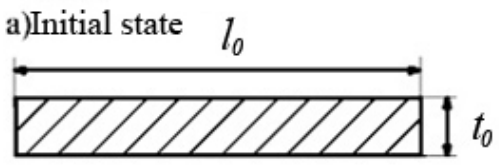

Cosine law : $t=t_{0} \cos \alpha$

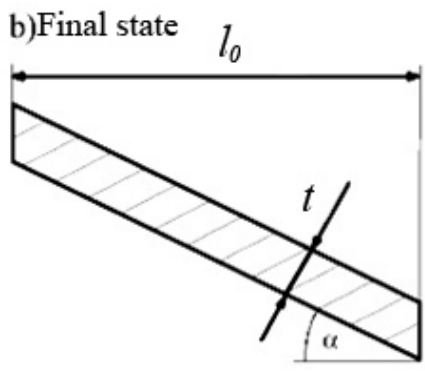

Fig. 4. The schematic of the cosine law.

\subsubsection{Study of overall shape}

In order to evaluate punch diameter effects on the overall geometry of formed sheet, the cross-section photographs of the sample were captured. Then, the final shape of the three regions of the formed sample has been detected and analyzed by image processing. The result will be presented in Section 3.2.

\subsubsection{Investigation of surface quality}

The forming process is occurred by applying the hammering punch to the clamped sheet. Based on the distance between the punch sequences, the roughness $R_{\mathrm{th}}$ is created on the surface of the sheet which is shown in Figure 5. This roughness is related to several parameters such as punch diameter, feed and the initial sheet radius.

By considering geometrical shape of surface, the relationships between effective parameters and the roughness can be found:

$$
f_{\mathrm{t}}=\sqrt{f_{\mathrm{h}}^{2}+f_{\mathrm{v}}^{2}}
$$

where $f_{\mathrm{h}}, f_{\mathrm{v}}$ and $f_{\mathrm{t}}$ are the horizontal, vertical and total feeds, respectively. Finally, the theoretical roughness was presented as following:

$$
R_{\mathrm{th}}= \begin{cases}R_{\mathrm{p}}-\sqrt{R_{\mathrm{p}}^{2}-f_{\mathrm{t}}^{2} / 4} \quad \text { Flat } & \\ R_{\mathrm{p}}-\sqrt{R_{\mathrm{p}}^{2}-f_{\mathrm{t}}^{2} / 4}+R_{\mathrm{s}}-\sqrt{R_{\mathrm{s}}^{2}-f_{\mathrm{t}}^{2} / 4} & \text { Convex } \\ R_{\mathrm{p}}-\sqrt{R_{\mathrm{p}}^{2}-f_{\mathrm{t}}^{2} / 4}-R_{\mathrm{s}}+\sqrt{R_{\mathrm{s}}^{2}-f_{\mathrm{t}}^{2} / 4} & \text { Concave }\end{cases}
$$

where $R_{\mathrm{p}}$ and $R_{\mathrm{s}}$ are the punch and initial sheet radius, respectively.

For experimental part of the work, several specimens were prepared in the radial direction for roughness measurement.

To evaluate the surface quality image processing was also used. Firstly, the photographs of several punched samples were captured. Then, according to the variation of light reflection for the different roughness values, the surface was investigated by the qualitative approach. As a result the variation of light reflection was changed to the sharp color. The result will be presented in Section 3.3.

\subsubsection{Evaluation of springback}

To evaluate the spring-back of the formed sheet, several rings are separated from the samples by wire-cut machine. The rings were separated at $10 \mathrm{~mm}$ height. Then, the deviation (from the initial state along $x$ and $y$ directions) was measured by cutting the rings. The general 

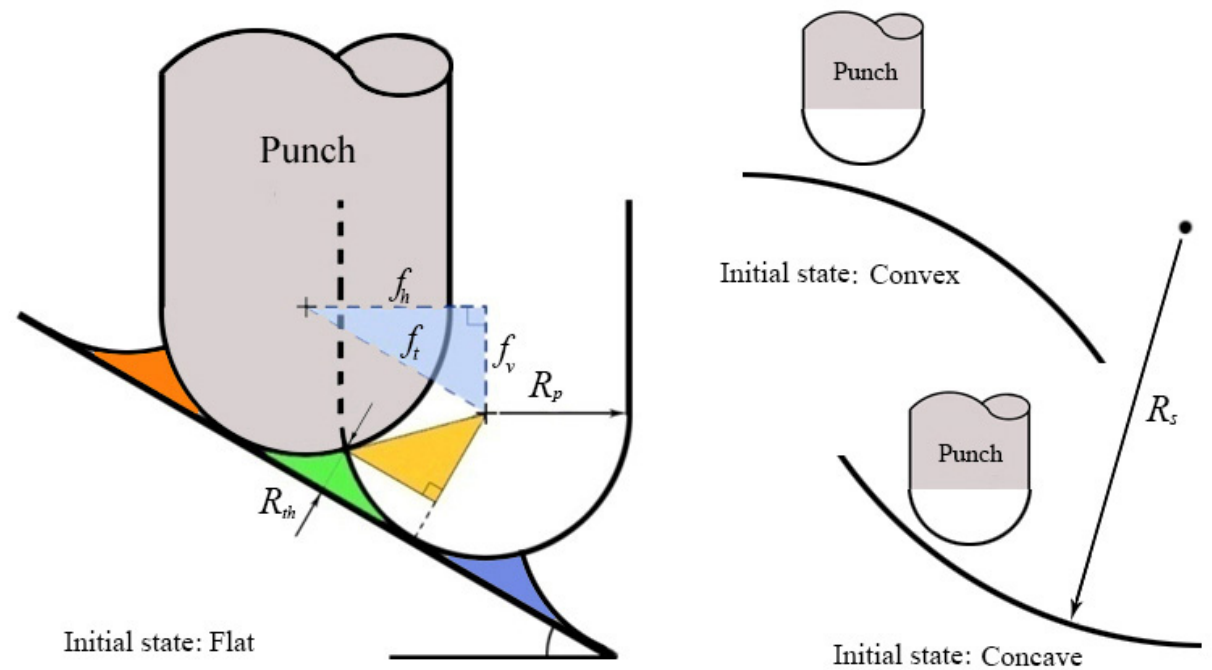

Fig. 5. Geometric study of the surface roughness.

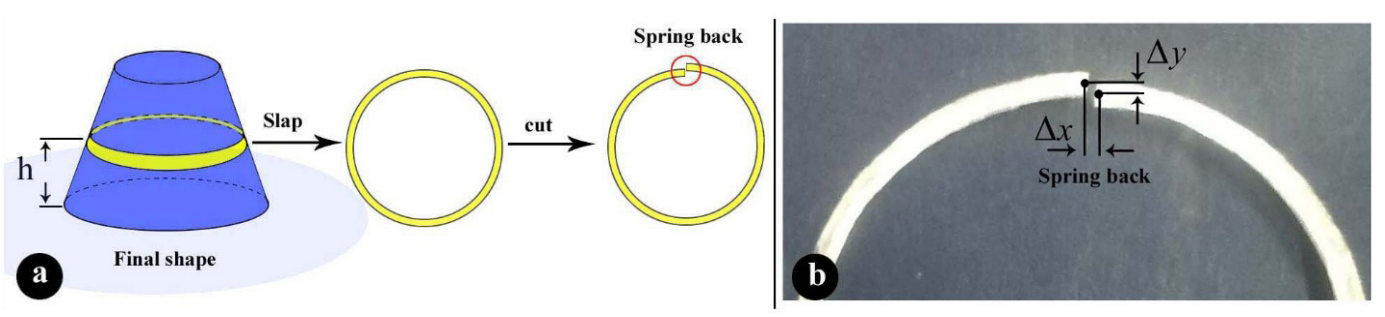

Fig. 6. The trend of spring-back measurement, (a) procedure to determine the spring-back, (b) observation of spring-back.

trend for the experiments is shown in Figure 6. The result will be presented in Section 3.4.

\section{Results and discussion}

In this section, the results of dimensional accuracy including final thickness, overall geometry, surface quality and the spring-back are presented.

\subsection{Final thickness}

The thickness variation in the cross-section of the samples which is achieved by image processing and cosine law is shown in Figure 7.

According to Figure 7, the cosine law has a good agreement with experiment for thickness variation. It shows that by reducing the punch diameter, the minimum thickness is moved toward lower part of cone wall. In fact, the forming mechanism for the incremental forming is divided into bending and stretching. When the punch diameter is decreased, sharper bending process occurred easily. So higher value of impact energy is consumed for stretching process and consequently the final thickness is decreased. As a result, a certain point on the sheet will be moved toward lower part of cone wall. Furthermore, punch is penetrated to the sheet by decreasing the punch diameter and the thickness is reduced again.

\subsection{Overall shape}

To evaluate the punch diameter effect on the overall shape, several specimens were punched by the hammering tool with three punch diameters $(D)$ of 3,5 and $10 \mathrm{~mm}$. The cross-section of the formed samples is shown in Figure 8.

According to Figure 8, the overall shapes have three critical regions which determine the accuracy and deviation from ideal shape. The amount of deviation in the region-A was increased by increasing the punch diameter. In fact, the variation of punch diameter had no effect on the cone wall. The region-B is one of the important regions in the overall geometry. The deviations of this area are declined by decreasing the punch diameter. The spring-back after forming process is the main cause for deviation of this area. The amount of deviation of $\mathrm{C}$ is known as pillow effect [11]. According to the figure, this effect was reduced by decreasing the punch diameter. The deviation of two regions $\mathrm{A}$ and $\mathrm{B}$ can be presented versus circle radius, that are tangent at the corners of the formed sheet. Also the variation of pillow effect was presented by parameter $L$ for region-C. The values of deviation for three regions of formed samples are presented in Table 1.

According to the table, the pillow effect was reduced about $32 \%$ by decreasing the punch diameter from 10 to $3 \mathrm{~mm}$. 

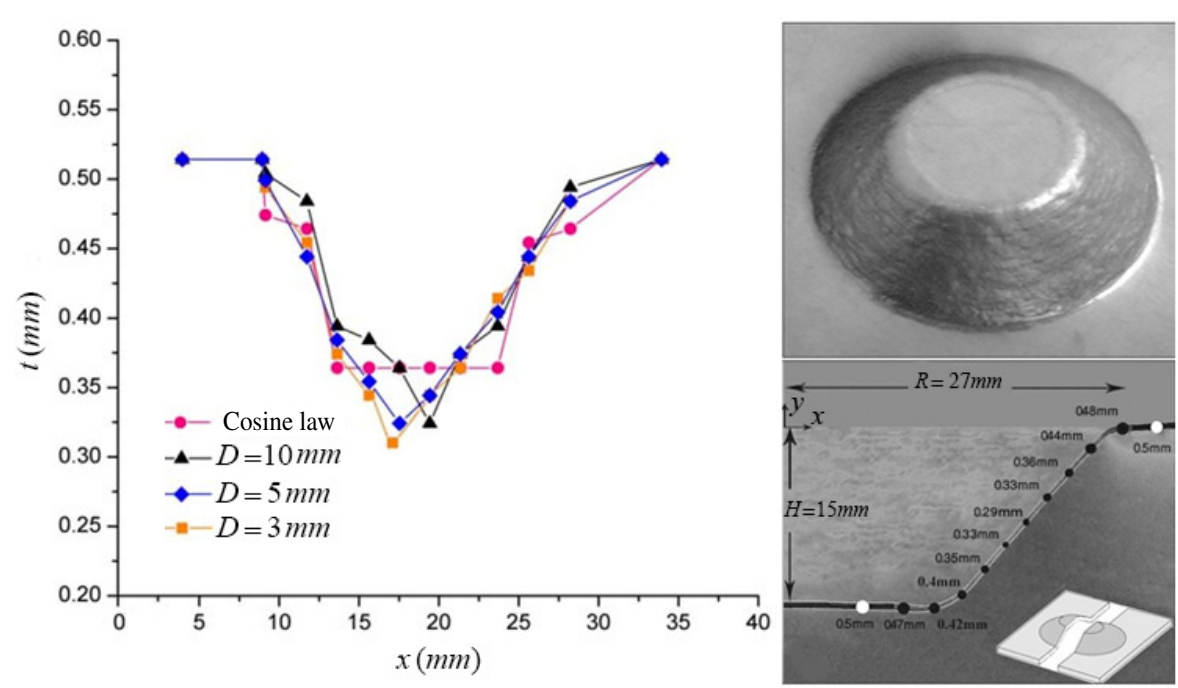

Fig. 7. The final thickness variation versus punch diameter $(D)$.

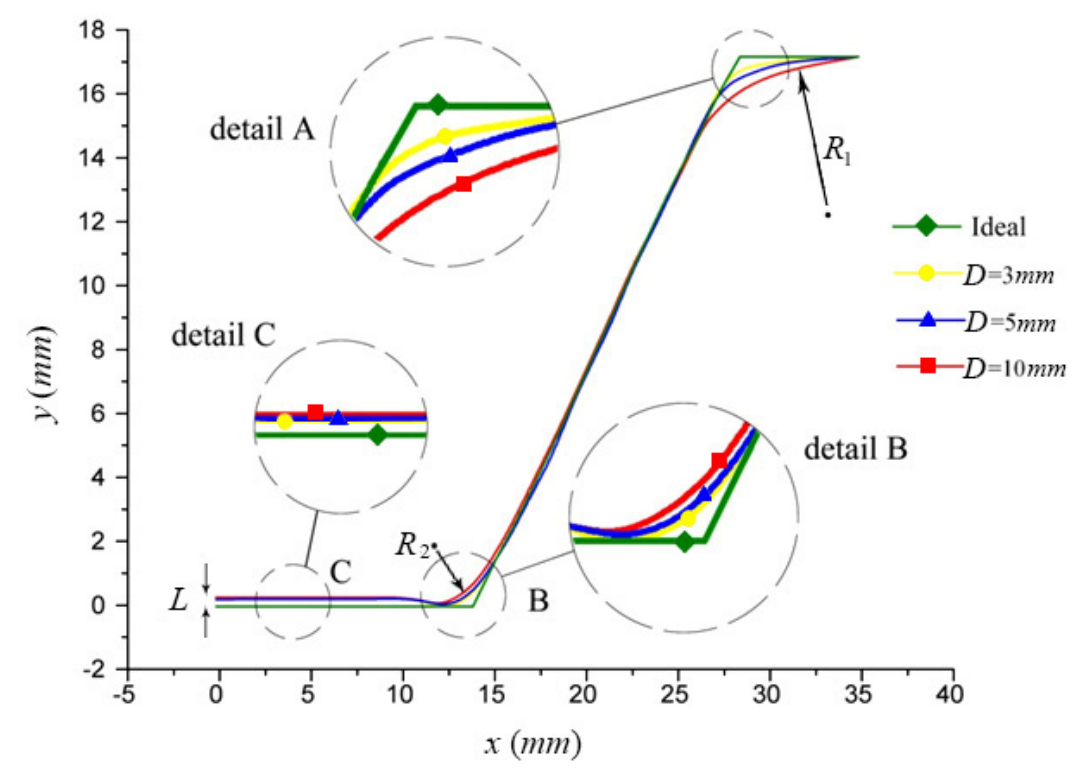

Fig. 8. Effect of punch diameter $(D)$ on the overall shape.

Table 1. The amount of deviations versus punch diameter $(D)$.

\begin{tabular}{cccc}
\hline$D(\mathrm{~mm})$ & $R_{1}(\mathrm{~mm})$ & $R_{2}(\mathrm{~mm})$ & $L(\mathrm{~mm})$ \\
\hline 10 & 6.08 & 2.6 & 0.25 \\
5 & 3.54 & 2.43 & 0.19 \\
3 & 1.58 & 2.31 & 0.17 \\
\hline
\end{tabular}

\subsection{Surface quality}

The samples roughness values were measured for three punch diameters of 3,5 and $10 \mathrm{~mm}$. The surface roughness measurement was accomplished for the inner and outer surface of formed sheet. The roughness value for the initial shape is $0.72 \mu \mathrm{m}$. The measured surface rough- ness is shown in Figure 9a. Also the detected roughness by image processing is shown in Figures $9 \mathrm{~b}$ and $9 \mathrm{c}$.

According to the Figure 9a, the roughness values were decreased by increasing the punch diameter. In addition, the theoretical values have a good validation with experimental results by increasing the punch diameter. The minor deviation of theoretical roughness for small punch diameter can be justified due to the material flow in the vicinity of punched areas (Fig. 10). As seen in Figures 9b and $9 \mathrm{c}$, the qualitative approach by image processing indicates that the surface quality for the $5 \mathrm{~mm}$ punch diameter is less than for $10 \mathrm{~mm}$ punch diameter. In fact, the variation of the color for the samples after image processing indicates the higher light reflection for small punch diameter. 

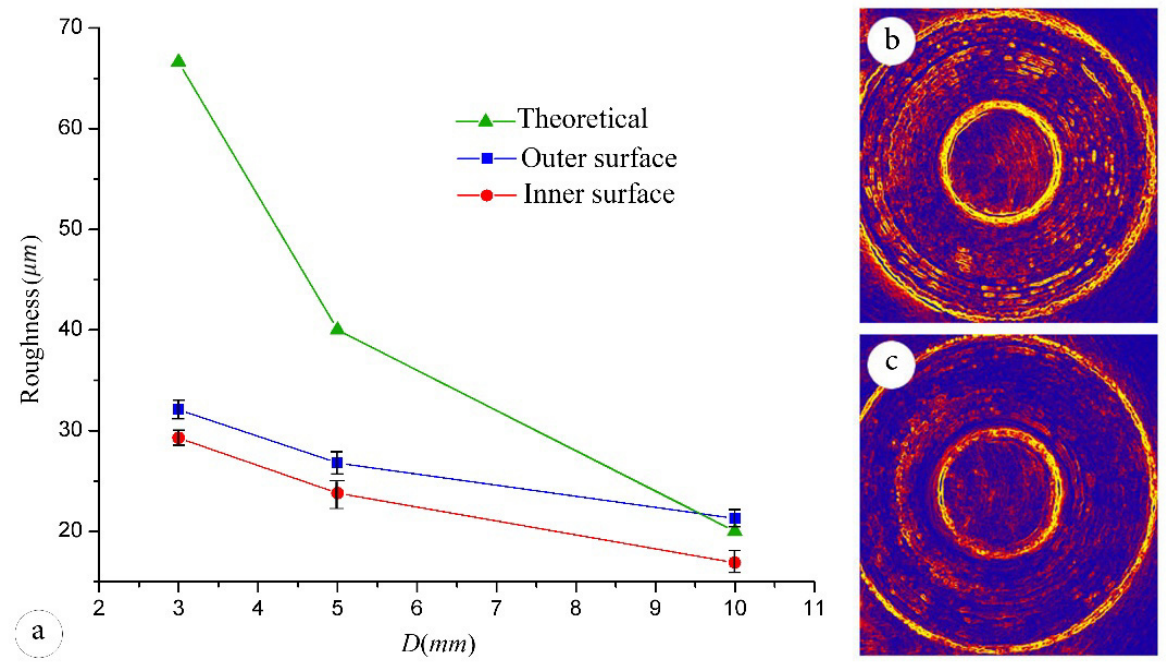

Fig. 9. The result of surface roughness, (a) roughness value for inner and outer surface and theoretical (flat) versus punch diameter $(D)$, (b) punch diameter of $5 \mathrm{~mm}$ and (c) punch diameter of $10 \mathrm{~mm}$.

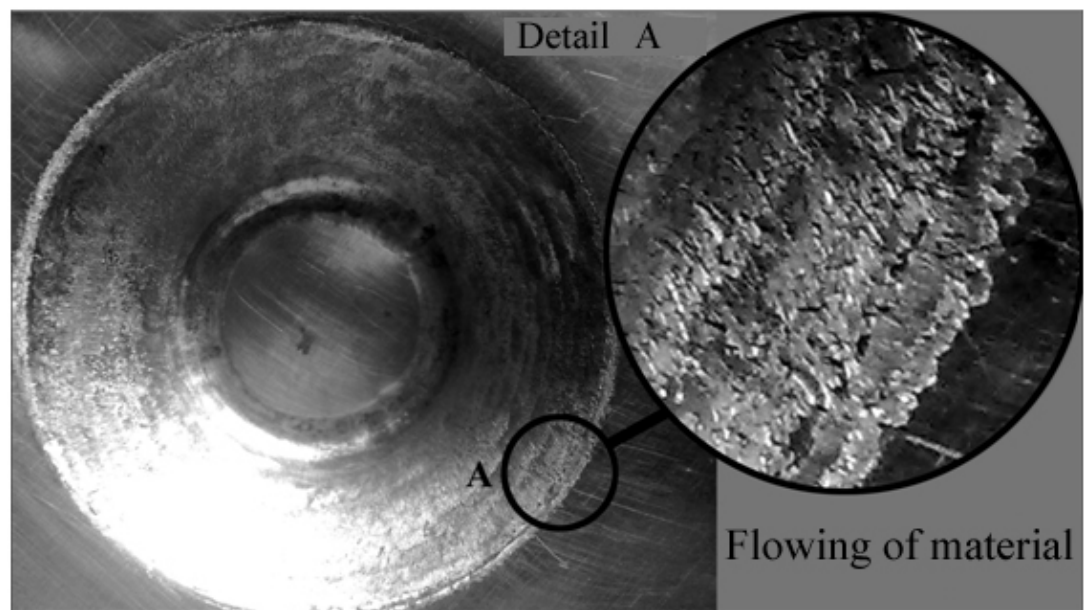

Fig. 10. View of the material flowing for small punch diameter $(5 \times$ magnifications $)$.

Table 2. The spring-back values versus punch diameter.

\begin{tabular}{ccc}
\hline$D(\mathrm{~mm})$ & $\Delta x(\mathrm{~mm})$ & $\Delta y(\mathrm{~mm})$ \\
\hline 3 & 0.32 & 0.2 \\
5 & 0.44 & 0.28 \\
10 & 0.52 & 0.34 \\
\hline
\end{tabular}

\subsection{Spring-back}

The spring-back values for the formed specimens with three punch diameters of 3,5 and $10 \mathrm{~mm}$ are presented in Table 2. This shows that the spring-back values in both $x$ and $y$ direction are reduced by decreasing the punch diameter.

\section{Conclusion}

In this paper, the effects of punch diameter on the dimensional accuracy in incremental sheet metal hammer- ing process (ISMH) were analyzed. The following conclusions can be highlighted:

- For evaluation of the dimensional accuracy in ISMH process, final thickness, overall geometry, surface quality and the spring-back variations are studied.

- It shows that by reducing the punch diameter, the minimum thickness is moved toward lower part of cone wall.

- The overall geometry had a smaller deviation than ideal state by decreasing the punch diameter. These deviations include increasing the curvature radius in the corner of cone shape and pillow effects.

- Surface roughness as a parameter of surface quality was improved with the increasing punch diameter. As a result, surface roughness was improved about $43 \%$ by increasing the punch diameter from 3 to $10 \mathrm{~mm}$.

- Spring-back values for both $x$ and $y$ directions were reduced by decreasing punch diameter. 


\section{References}

[1] N. Nakajima, Numerical control for traditional manual forming of sheet metal, J. Japan Society Technol. Plasticity 23 (1979) 696-700

[2] J. Allwood, H. Utsunomiya, A survey of flexible forming processes in Japan, Int. J. Mach. Tools Manuf. 46 (2006) $1939-1960$

[3] G. Ambrogio, I. Costantino, L. De Napoli, L. Filice, L. Fratini, M. Muzzupappa, Influence of some relevant parameters on the dimensional accuracy in incremental forming: a numerical and experimental investigation, J. Mater. Process. Technol. 153 (2004) 153-154

[4] F. Micari, G. Ambrogio, L. Filice, Shape and dimensional accuracy in Single Point Incremental Forming: State of the art and future trends, J. Mater. Process. Technol. 191 (2007) 390-395

[5] F. Han, J. Mo, H. Wei, R. Fen, X. Hui, Z. Wei, Spring back prediction for incremental sheet forming Based on FEM-PSONN technology, Trans. Nonferrous Metals Soc. China 23 (2013) 1061-1071
[6] M. Ham, J. Jeswiet, Dimensional Accuracy Of Single point incremental forming, Int. J. Mater. Forming 1 (2008) 1171-1174

[7] A. Puzik, Incremental sheet forming with a robot system for an industrial application, 41st CIRP Conference on Manufacturing Systems, 2008

[8] T. Schafer, R. Scraft, Incremental sheet metal forming by industrial robots, Rapid Prototyping J. 5 (2005) 278-286

[9] H. Tanaka, N. Asakawa, M. Hirao, Forming type rapid prototyping development - error compensation with shape measurement, J. Automation Technol. 2 (2008) 462-467

[10] Y. Luo, K. He, R. Du, A new sheet metal forming system based on the incremental Punching, part 1: modeling and simulation, Int. J. Adv. Manuf. Technol. 51 (2010) 481-491

[11] G. Ambrogio, V. Cozza, L. Filice, F. Micari, An analytical model for improving precision in single point incremental forming, J. Mater. Process. Technol. 191 (2007) 92-95 